\title{
CHARACTERIZATIONS OF MEASURES WHOSE FOURIER-STIELTJES TRANSFORMS VANISH AT INFINITY
}

\author{
BY RUSSELL LYONS ${ }^{1}$
}

We denote the class of complex Borel measures on the unit circle $\mathbf{T}=\mathbf{R} / \mathbf{Z}$ by $M(\mathrm{~T})$. Those measures $\mu$ whose Fourier-Stieltjes coefficients

$$
\hat{\mu}(n)=\int_{\mathbf{T}} e^{-2 \pi i n t} d \mu(t)
$$

tend to 0 as $|n| \rightarrow \infty$ form the class $R$. The class of Borel sets $E$ such that $\mu E=0$ for all $\mu \in R$ is denoted $U_{0}$. We announce relations of $R$ to certain subclasses of $U_{0}$. Full proofs will appear elsewhere.

Theorem 1. A measure $\mu$ lies in $R$ if and only if $\mu E=0$ for all $E \in U_{0}$.

This result is a corollary of our principal result, Theorem 3 below. Let $J$ be the class of measures concentrated on a $U_{0}$-set. An immediate consequence of Theorem 1 is

CoROLlaRY 2. Each measure $\mu \in M(\mathbf{T})$ can be uniquely represented as $\mu=\mu_{R}+\mu_{J}$, where $\mu_{R} \in R, \mu_{J} \in J$, and $\mu_{R} \perp \mu_{J}$.

Our method of proof of Theorem 1 involves a subclass of $U_{0}$, called $W$-sets. We first recall some facts from the theory of asymptotic distribution.

Definition. A sequence $\left\{x_{n}\right\}_{1}^{\infty} \subset \mathbf{T}$ is said to have an asymptotic distribution if there exists $\nu \in M(\mathrm{~T})$ such that for every $\operatorname{arc} I \subset \mathbf{T}$ whose endpoints are not mass-points of $\nu$,

$$
\lim _{N \rightarrow \infty} \frac{1}{N} \#\left\{n \leq N: x_{n} \in I\right\}=\nu I \text {. }
$$

In this case, $\nu$ is called the asymptotic distribution of $\left\{x_{n}\right\}$. If $\nu$ is normalized Lebesgue measure, then $\left\{x_{n}\right\}$ is said to be uniformly distributed.

The principal theorem associated with asymptotic distribution usually goes by the name of "Weyl's criterion" (see [6, I, p. 142] for a proof).

Weyl's CRIterion. A sequence $\left\{x_{n}\right\}_{1}^{\infty} \subset \mathbf{T}$ has an asymptotic distribution if and only if for every $m \in \mathbf{Z}$,

$$
\lim _{N \rightarrow \infty} \frac{1}{N} \sum_{n=1}^{N} e^{-2 \pi i m x_{n}} \text { exists. }
$$

In this case, the limit is $\hat{\nu}(m)$, where $\nu$ is the asymptotic distribution.

Received by the editors May 25, 1983.

1980 Mathematics Subject Classification. Primary 43A25; Secondary 43A10, 42A63, 43A46, 42A55, 10K05, 10K25, $10 \mathrm{~K} 50$.

${ }^{1}$ This research was supported in part by an NSF Graduate Fellowship and by NSF Grant MCS 82-01602. 
DEFINITION [5]. If $\left\{x_{n}\right\}$ has an asymptotic distribution but is not uniformly distributed, then we say $\left\{x_{n}\right\}$ is Weyl-distributed. A Borel set $E \subset \mathbf{T}$ is called a $W$-set if there exists a strictly increasing sequence of positive integers $\left\{n_{k}\right\}$ such that for every $x \in E,\left\{n_{k} x\right\}$ is Weyl-distributed.

SreY̌der [5] made the following unproved claim, which we have established.

TheOREM 3. A measure $\mu$ lies in $R$ if and only if $\mu E=0$ for all $W$-sets $E$.

The proof of Theorem 3 depends on Weyl's criterion and the well-known fact [6, II, p. 145] that if $\mu \in R$ and $|\nu|$ is absolutely continuous with respect to $|\mu|$, then $\nu \in R$. The crucial ingredient of our proof, however, is a new remarkable lemma.

LEMMA 4. Given $\mu \in M(\mathbf{T})$ and $\left\{n_{k}\right\}_{1}^{\infty} \subset \mathbf{Z}$, there exists a subsequence $\left\{n_{k}^{\prime}\right\} \subset\left\{n_{k}\right\}$ such that $\left\{n_{k}^{\prime} x\right\}$ has an asymptotic distribution for $|\mu|$-almost all $x$.

A sketch of the proof of Lemma 4 follows. Choose a subsequence $\left\{e^{-2 \pi i n_{k}^{\prime \prime} x}\right\}$ of $\left\{e^{-2 \pi i n_{k} x}\right\}$ which weakly converges in $L^{2}(|\mu|)$. We may find a further subsequence $\left\{e^{-2 \pi i n_{k}^{\prime} x}\right\}$ such that if $f(x)$ is the weak limit of $e^{-2 \pi i n_{k}^{\prime \prime} x}$, then

$$
\left\|\frac{1}{K} \sum_{k=1}^{K} e^{-2 \pi i n_{k}^{\prime} x}-f\right\|_{L^{2}(|\mu|)}=O\left(K^{-1 / 2}\right) .
$$

It follows that

$$
\frac{1}{K^{2}} \sum_{1}^{K^{2}} e^{-2 \pi i n_{k}^{\prime} x} \rightarrow f(x) \text { a.e }[|\mu|]
$$

as $K \rightarrow \infty$, from which we deduce that also

$$
\frac{1}{K} \sum_{1}^{K} e^{-2 \pi i n_{k}^{\prime} x} \rightarrow f(x) \text { a.e. }[|\mu|] .
$$

By a diagonal argument, we may choose $n_{k}^{\prime}$ so that, likewise, for every $m \in \mathbf{Z}$,

$$
\lim _{K \rightarrow \infty} \frac{1}{K} \sum_{1}^{K} e^{-2 \pi i m n_{k}^{\prime} x} \text { exists a.e. }[|\mu|] .
$$

Weyl's criterion now implies that $\left\{n_{k}^{\prime} x\right\}$ has an asymptotic distribution for $|\mu|$-a.e. $x$.

Theorem 3 extends in a natural way to all locally compact abelian groups.

Other attempts have been made in the past to characterize $R$ by a theorem of the form of Theorem 3. Rajchman had conjectured (see [2, pp. 85-86]) that the class of $H$-sets, defined below, could be taken in place of the class of $W$-sets. On the other hand, Kahane and Salem $[3,4]$ asked whether $U_{0}$ contains the class of $W^{*}$-sets, to be defined presently. As we shall see, this amounts to the question of whether $W^{*}$-sets can replace $W$-sets in Theorem 3. However, neither $H$-sets nor $W^{*}$-sets can replace $W$-sets (see Theorems 7, 6 below).

Definition. A Borel set $E \subset \mathbf{T}$ is a $W^{*}$-set (or nonnormal set) if there exists $n_{k} \uparrow \infty$ such that for every $x \in E,\left\{n_{k} x\right\}$ is not uniformly distributed.

Since $W$-sets are clearly $W^{*}$-sets, Theorem 3 implies 
Theorem 5. If $\mu E=0$ for all $E \in W^{*}$, then $\mu \in R$.

Actually, Theorem 5 has an elementary proof. Briefly, if $\mu \notin R$, take $\hat{\mu}\left(n_{k}\right) \rightarrow \alpha \neq 0$. Let

$$
E=\left\{x:\left\{n_{k} x\right\} \text { is not uniformly distributed }\right\} .
$$

Then one may show that

$$
\lim _{K \rightarrow \infty} \int_{E} \frac{1}{K} \sum_{k=1}^{K} e^{-2 \pi i n_{k} x} d \mu(x)=\alpha \neq 0,
$$

whence $|\mu| E \neq 0$. Since $E$ is a $W^{*}$-set, Theorem 5 follows. We remark that the ideas here are those necessary to prove Theorem 3 using Lemma 4.

Thus, we see that Kahane's and Salem's question of whether $W^{*} \subset U_{0}$ is equivalent to asking if $W^{*}$-sets characterize $R$ in the manner that $W$-sets do. As we have said, this is untrue. We may, in fact, give precise conditions on the rate of decay of $\hat{\mu}$ at infinity in order that $\mu$ annihilate all $W^{*}$-sets corresponding to a sequence $\left\{n_{k}\right\}$ with $\liminf _{k \rightarrow \infty} n_{k+1} / n_{k}>1$, the so-called lacunary $W^{*}$-sets.

THEOREM 6. If $\phi(n)$ is a decreasing function on the nonnegative integers such that

$$
\sum_{n=2}^{\infty} \frac{\phi(n)}{n \log n}<\infty,
$$

and if $\mu$ is a positive measure such that

$$
|\hat{\mu}(n)| \leq \phi(|n|)
$$

for all $n$, then $\mu E=0$ for all lacunary $W^{*}$-sets. However, if $\phi$ is decreasing and (1) fails, then there exists a positive measure $\mu$ satisfying (2) and such that $\mu$ is concentrated on the set of nonnormal numbers base 2 , i.e., on the lacunary $W^{*}$-set

$$
E=\left\{x \in \mathbf{T}:\left\{2^{k-1} x\right\}_{k=1}^{\infty} \text { is not uniformly distributed }\right\}
$$

The first part of Theorem 6 is a slight extension of a result of Baker [1]. The proof of the second part involves infinite convolution measures.

We now turn to Rajchman's conjecture about $H$-sets.

Definition. A Borel set $E \subset \mathbf{T}$ is called an $H$-set if there exists $n_{k} \uparrow \infty$ and a nonempty open arc $I \subset \mathbf{T}$ such that for all $x \in E$ and all $k, n_{k} x \notin I$.

It has long been known that $H \subset U_{0}$. Rajchman's conjecture asserts that also $\mu \in R$ if $\mu E=0$ for all $H$-sets $E$. A counterexample to this assertion is given by the next result.

TheOREM 7. Let $\mu$ be the Riesz product

$$
d \mu=\prod_{k=1}^{\infty}\left(1+a_{k} \cos 2 \pi\left(n_{k} x+\phi_{k}\right)\right) d x
$$

where $-1 \leq a_{k} \leq 1$. If $n_{k+1} / n_{k} \rightarrow \infty$, then $\mu E=0$ for all $H$-sets $E$. 
Note that if $a_{k} \neq 0$, then $\mu \notin R$. It can also be shown that Riesz products not belonging to $R$ are concentrated on $W$-sets and hence belong to $J$. Thus, Riesz products $\mu$ are pure: $\mu \in R$ or $\mu \in J$. The same holds for infinite convolutions of discrete measures, extending the Jessen-Wintner purity law.

Extensive discussions with Professor Hugh L. Montgomery have been invaluable in attaining these results. The author is also indebted to Professor Allen L. Shields for raising the question of whether Theorem 1 is true and for giving reference [5].

\section{REFERENCES}

1. R. C. Baker, A diophantine problem on groups. IV, Illinois J. Math. 18 (1974), 552-564.

2. N. K. Bari, The uniqueness problem of the representation of functions by a trigonometric series, Amer. Math. Soc. Transl. No. 52, (1951), 1-89.

3. J.-P. Kahane, Sur les mauvaises répartitions modulo 1, Ann. Inst. Fourier (Grenoble) 14 (1964), 519-526.

4. J.-P. Kahane and R. Salem, Distribution modulo 1 and sets of uniqueness, Bull. Amer. Math. Soc. 70 (1964), 259-261.

5. Ju. A. Sreřder, On the Fourier-Stieltjes coefficients of functions with bounded variation, Dokl. Akad. Nauk SSSR 74 (1950), 663-664. (Russian)

6. A. Zygmund, Trigonometric series, 2nd ed., reprinted, Vols. I, II, Cambridge Univ. Press, Cambridge, 1979.

Department of Mathematics, University of Michigan, Ann Arbor, Michigan 48109

Current address: Bâtiment de Mathématique, No. 425, Université de Paris-Sud, 91405 Orsay, France 